\title{
Role of Computed Tomography (CT) Enterography in the Evaluation of Small Bowel Diseases
}

\author{
Fawaz Yousuf ${ }^{1}$, Sanjay Sethi², Ranjana Gupta ${ }^{3}$, Sandeep Joshi ${ }^{4}$, Puneet Mittal ${ }^{5}$, Divya Nijhawan ${ }^{6}$ \\ 1, 2, 3, 5, 6 Department of Radiodiagnosis, MM Institute of Medical Sciences \& Research, Mullana, Ambala, Haryana, India. \\ ${ }^{4}$ Department of Medicine, MM Institute of Medical Sciences \& Research, Mullana, Ambala, Haryana, India.
}

\section{ABSTRACT}

\section{BACKGROUND}

Small bowel imaging often questions clinicians due to its long, tortuous and undulating morphology. The current study was undertaken to establish the efficacy of multidetector computed tomography (MDCT) enterography in the evaluation of small bowel diseases using iso-osmotic mannitol as oral contrast agent.

\section{METHODS}

A prospective observational study was conducted among thirty patients (mean age 40 years; range 14 - 66 years), 16 males and 14 females presenting with clinical suspicion of small bowel diseases based on clinical, laboratory or other imaging investigations underwent CT enterography on a 128 slice CT scanner using isoosmotic mannitol as neutral enteral contrast.

\section{RESULTS}

Intestinal tuberculosis was the most common diagnosis. Non neoplastic cases predominantly showed segmental symmetrical involvement with target pattern of enhancement. Small bowel tumours (adenocarcinoma) were seen in 2 cases. Neoplasms were associated with focal asymmetrical involvement with heterogeneous enhancement pattern. Computerized tomography enterography (CTE) clearly depicted the site, level and cause of the obstruction in all the patients with a sensitivity and specificity of $96 \%$ and $100 \%$ respectively. Diagnostic accuracy of CTE in detection of small bowel diseases came out to be $98 \%$.

\section{CONCLUSIONS}

CT enterography is an effectual, non-invasive, well-tolerated, sensitive and specific imaging modality for the evaluation of small bowel diseases which provides superior assessment of the intraluminal, mural and extraintestinal pathologies.

\section{KEY WORDS}

CT enterography, mannitol, Tuberculosis, Small Bowel
Corresponding Author: Dr. Sanjay Sethi,

41, Urban Estate, Phase-2, Patiala - 147002, Haryana, India.

E-mail: sanaanshu2012@gmail.com

DOI: $10.14260 / j e m d s / 2021 / 305$

How to Cite This Article:

Yousuf F, Sethi S, Gupta R, et al. Role of computed topography (CT) enterography in the evaluation of small bowel diseases. $J$ Evolution Med Dent Sci 2021;10(20):14511456, DOI: $10.14260 / \mathrm{jemds} / 2021 / 305$

Submission 08-01-2021,

Peer Review 16-03-2021

Acceptance 23-03-2021,

Published 17-05-2021.

Copyright (c) 2021 Fawaz Yousuf et al. This is an open access article distributed under Creative Commons Attribution License [Attribution 4.0 International (CC BY 4.0)] 


\section{BACKGROUND}

Small bowel imaging is often tedious task because of its length, tortuosity and the undulating bowel. However, its imaging has been improvised with upcoming advancements. Traditional small bowel follow through examinations are nowadays being replaced by cross sectional imaging. ${ }^{1}$

CT enterography, an emerging non-invasive modality for imaging the small bowel combines the advantages of temporal and spatial resolution offered by MDCT scanners with optimal visualisation of the enteric mucosa and bowel wall ensured by the oral and intravenous contrast agents. It is well tolerated by patients. However, the drawbacks of CT enterography are usage of I.V contrast and repeated exposure to ionizing radiations, the latter being an advantage of magnetic resonance (MR) enterography over CT which has caused MR enterography to be favoured among younger patients with inflammatory bowel disease that warrant follow up imaging. 2,3 The contrast material used in MRE has lesser incidence of allergic reaction than iodine-based contrast used in conventional x-rays and CT. However, the limitations of MR enterography are its high cost, time consumption and variation in image quality. CTE is preferred over magnetic resonance enterography (MRE) when patient has trouble holding their breath and for ensuring superior spatial resolution and high quality multiplanar reformation.

Though fluoroscopic studies like barium meal follow through (BMFT) ensures luminal distension hence making it ideal for viewing the enteric mucosa and partial strictures, the overlapping bowel reduces the sensitivity of the test. Even capsule endoscopy has superior mucosal visualisation and abilities to obtain biopsies for assessing histologically, but is often limited by its inability to view extra enteric structures, subjective interpretation, difficulty in tumour localisation and its usage in patients having strictures due to its chances of getting lodged within. ${ }^{1,4}$ CT and MR enteroclysis also works on the principles of luminal distension with enteric contrast material through a nasoenteric tube followed by spatial and temporal viewing of the bowel. It is an ideal modality for detecting strictures. However; the semi-invasiveness causing patient discomfort and expert operator dependency often restricts their widespread use. ${ }^{5}$

Imaging with computerised tomography (CT) with enteroclysis / enterography (CTE) or magnetic resonance enteroclysis has become an essential tool because of their capability of disease localisation as well as evaluating the entire intestinal wall thickness, mesentery and lymph nodes. ${ }^{6}$ This study was conducted to evaluate the role of multi-slice CT enterography in different bowel diseases in patients and to describe CT enterography findings in commonly encountered pathologies.

\section{METHODS}

The present study was conducted in the Department of Radiodiagnosis, Maharishi Markandeshwar Institute of Medical Sciences and Research (MMIMSR), Mullana from January 2019 to September 2020. A prospective observational study was conducted on thirty patients presenting to MMIMSR and referred to the Department of Radiodiagnosis for CT enterography with suspicion of small bowel diseases based on clinical, laboratory or other imaging investigations. All adult patients between 14 - 66 years of either sex, satisfying the selection criteria were recruited in the study.

\section{Inclusion Criteria}

Clinical suspicion of small bowel pathology. Patients with history of abdominal pain, features of small bowel obstruction, vomiting and chronic diarrhoea were included in the study.

\section{Exclusion Criteria}

1. Patient refusing to give consent.

2. Pregnancy.

3. Documented allergy to iodinated contrast.

4. Debilitated patients unable to drink large volume of fluid.

5. Post-surgical patients with ileostomy.

6. Comorbid conditions where fluid overload is to be avoided (patients suffering from cardiac, renal diseases).

7. Hemodynamically unstable patients with GI bleed.

8. Deranged blood urea and serum creatinine.

History of the patients was recorded regarding age, sex, any family history of similar diseases. All eligible patients were properly counselled and explained about the nature and purpose of the study. Relevant investigations were done.

\section{Technique of Examination}

- Preparation and administration of oral contrast: $20 \%$ of $450 \mathrm{ml}$ of mannitol was mixed with $1350 \mathrm{ml}$ of water to make it $1800 \mathrm{ml}$ of iso-osmotic mannitol.

- The entire volume of oral contrast was offered to the patient and asked to drink under supervision as follows:

a) $500 \mathrm{ml}$ in each $20 \mathrm{~min}$ for an hour duration.

b) A table dose of $300 \mathrm{ml}$ was given to distend the stomach just before scanning.

c) Amount of the oral contrast intake was recorded.

d) Any problem due to contrast was noted.

- Acquisition of images: CT enterography examination was conducted.

Acquisition protocol for MDCT enterography:

- Scanning extent: Diaphragm to the pubic symphysis.

- $\quad \mathrm{kVp}: 120 \mathrm{kVp}$

- Effective mAs: $251 \mathrm{mAs}$

- Detector collimation: $64 \times 0.625$

- I.V contrast (non-ionic contrast): $350 \mathrm{mg} / \mathrm{ml}$ Omnipaque

- Acquisition time: Enteric phase - 45 seconds after starting of scan.

- Images were sent and reviewed at a dedicated work station. Multiplanar and maximum intensity projection (MIP) reconstruction was done.

- Following non-contrast computed tomography (NCCT), contrast-enhanced computed tomography (CECT) was acquired from the level of diaphragm to pubic symphysis. CECT was acquired during the enteric phase of contrast enhancement for all the patients.

- Non-ionic iodinated contrast media (350 $\mathrm{mg} \%$ of Omnipaque) was given intravenously (I.V). Amount of I.V contrast given ranged from $80-120 \mathrm{ml}$ depending on the weight of the patient. 


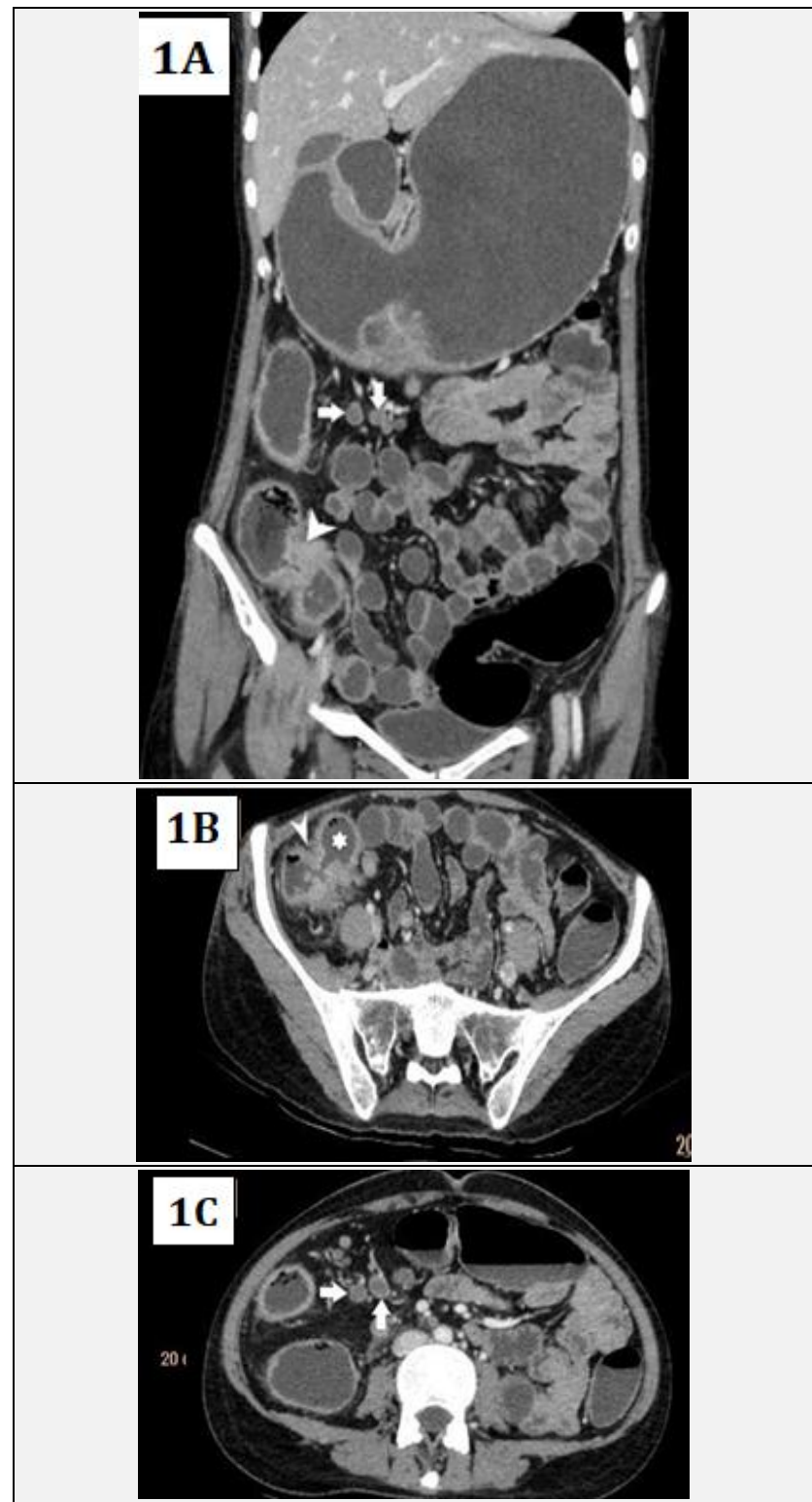

Figure 1. CT Enterography Coronal (1A) and Axial (1B \& 1C) Images Showing Symmetric Circumferential Mural Thickening of the Terminal Ileum (Arrowheads) Involving the IC Junction with Mild Prominence of Upstream Distal Ileum (Asterisk) with Necrotic Lymph Nodes (Arrows in $1 A$ and $1 C$ ) in a Patient with Intestinal Tuberculosis

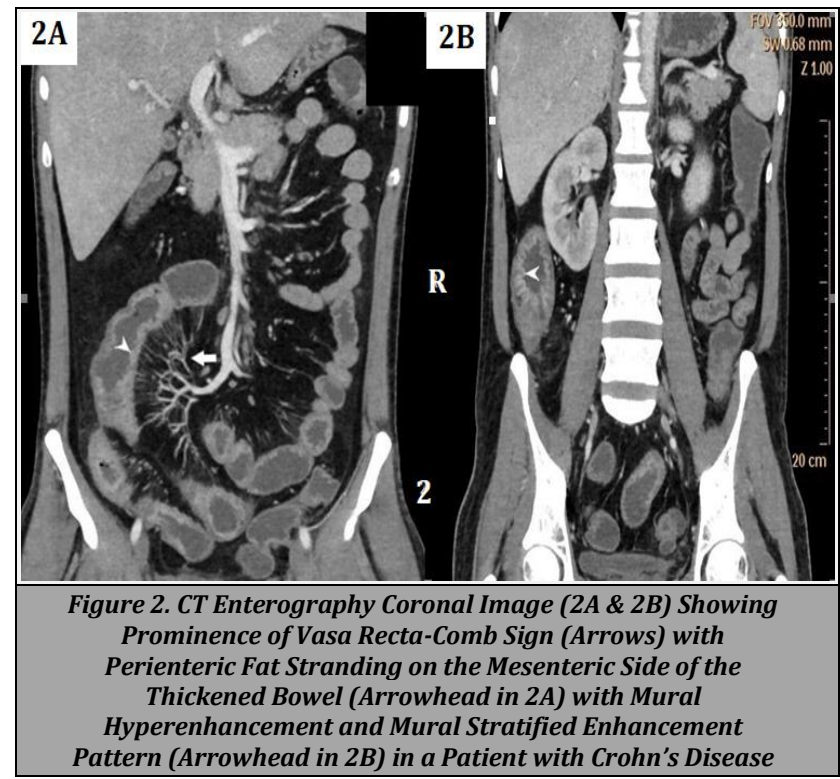

- $\quad$ After the acquisition of CT enterography, the patient was kept under observation for 4 hours and any diarrhoea or adverse effects of oral contrast were noted. The various side effects of the oral iso-osmotic mannitol were recorded.

- 2 days post enterography the patient was interviewed telephonically regarding any side effect due oral intake of mannitol.

\section{Image Recording and Evaluation}

1. The feasibility of CT enterography using iso-osmotic mannitol as a neutral oral contrast agent was assessed by evaluating its performance for distension of the entire small bowel.

2. The adequacy of small bowel distension was assessed as Grade III, Grade II or Grade I.

3. The overall small bowel evaluation in each study was graded as good, fair or bad.

4. Details of small bowel lesion and extra intestinal strictures and other abdominal organs were evaluated.

\section{Statistical Analysis}

'The recorded data was compiled. The sensitivity, specificity, positive and negative predictive value of CT enterography was calculated with respect to final diagnosis.

\section{RESULTS}

Out of 30 patients, females were $14(46.7 \%)$ and males were 16 (53.3\%). The mean age of our study was $40+/-16$ years. Most of our patients (23.3\%) were in the age group of $41-50$ years. The feasibility of CT enterography was evaluated on the basis of complete visibility of the various intestinal segments via adequate distension. Grading was done and collapsed bowel loop was graded as Grade I; bowel loops less than 1.5 $\mathrm{cm}$ in diameter with incomplete fold visibility was Grade II; and bowel distention more than $1.5 \mathrm{~cm}$ with complete fold visibility was graded as Grade III. Measurements were taken, at the level of superior mesenteric artery for jejunum and common iliac bifurcation for ileum. Majority (76.67 \%) of the patients showed adequate Grade III distension of both the jejunal and ileal loops. Grade II distensibility of the jejunal loops and ileal loops were seen in $23.3 \%$ of the cases.

Bowel thickening and mural hyperenhancement was the most common CT enterography finding, present in 23 (76.7\%) cases. Significant lymphadenopathy was present in 12 (40\%) cases. Engorged vasa recta were seen in 10 (33.33\%) patients. Bowel was dilated in 10 (33.3\%) cases. Ascites was seen in 10 $(33.3 \%)$ of the patients. Skip lesions were present in 6 (20\%) cases. Strictures were seen in $5(16.6 \%)$ cases. 2 (6.7\%) patients each had fistula and adhesions.

According to table, jejunal and ileal segments of the small intestine and the colon showed involvement in isolation or combinations, with ileum alone affected in 7 (23.3\%) cases. In $6(20 \%)$ cases both ileum and colon were involved. $5(16.6 \%)$ cases had no visible pathology. 4 (13.3\%) patients each had pathologies involving the jejunum, ileum and jejunum, ileum and colon respectively. 


\begin{tabular}{|c|c|c|c|c|c|}
\hline & $\begin{array}{c}\text { CTE Dx } \\
\text { Frequency }\end{array}$ & Percentage & & $\begin{array}{c}\text { Final Dx } \\
\text { Frequency }\end{array}$ & Percentage \\
\hline Adhesive band & 2 & 6.7 & Adhesive band & 2 & 6.7 \\
\hline Crohn's disease & 3 & 10.0 & Crohn's disease & 6 & 20 \\
\hline Celiac dis ease & 1 & 3.3 & Celiac disease & 1 & 3.3 \\
\hline Ischemic stricture & 2 & 6.7 & Ischemic stricture & 2 & 6.7 \\
\hline Radiation enteritis & 1 & 3.3 & Radiation enteritis & 1 & 3.3 \\
\hline Tuberculosis & 7 & 23.3 & Tuberculosis & 9 & 30 \\
\hline Adenocarcinoma & 2 & 6.7 & Adenocarcinoma & 2 & 6.7 \\
\hline Ulcerative colitis & 2 & 6.7 & Ulcerative colitis & 3 & 10.0 \\
\hline Normal & 5 & 16.7 & Normal & 4 & 13.3 \\
\hline Strictures / inflammation / nature & 1 & 3.3 & Total & 30 & 100.0 \\
\hline Partial stricture / tuberculosis & 1 & 3.3 & & & \\
\hline Crohn's disease / tuberculosis & 3 & 10.0 & & & \\
\hline Total & 30 & 100.0 & & & \\
\hline
\end{tabular}

In $2(6.7 \%)$ patients the colon alone was involved. 1 (3.3 $\%)$ patient each had isolated jejunal and a combined involvement of jejunum and colon respectively. Bowel wall thickening was seen in 23 subjects which was symmetrical in 19 and asymmetrical in 4 subjects. Abnormal mural enhancement was seen in 23 subjects. 16 (69.56\%) of them displayed a target pattern. Homogeneous enhancement was seen in 5 (21.72\%) cases with 2 (8.69\%) patients having heterogeneous mural enhancement.

In our study, CTE diagnosis for the small bowel pathologies included 23 non-malignant inflammatory cases and 2 malignant cases. 7 (23.3\%) cases of ileo-caecal tuberculosis (ITB), 3 (10 \%) cases of Crohn's disease (CD). 3 (10\%) cases showed signs of both $\mathrm{CD}$ and ITB which could not be confirmed on CTE. 2 (6.7\%) cases each of ulcerative colitis (UC), ischemic strictures and adhesive bands respectively. 1 (3.3\%) case each of radiation enteritis, celiac disease and partial strictures. 2 malignant cases of adenocarcinoma were diagnosed. In the final diagnosis which was made after compiling and comparing adequate laboratory, endoscopic and histopathologic proof of diagnosis, included 24 non-malignant inflammatory cases with 9 (30\%) cases of ITB, 6 (20\%) cases of CD. 3 (10\%) cases of UC. 2 (6.7 \%) cases each of ischemic strictures and adhesive bands. 1 (3.3\%) case each of radiation enteritis and celiac disease. 2 malignant cases of adenocarcinoma were diagnosed. (Table 1).

Based on the comparison of the CTE and final diagnosis the sensitivity, the specificity, accuracy, the PPV and NPV were calculated. CTE clearly depicted site, level and cause of the obstruction in all the patients with a sensitivity and specificity of $96 \%$ and $100 \%$ respectively. Diagnostic accuracy of CTE in detection of small bowel diseases came out to be $98 \%$.

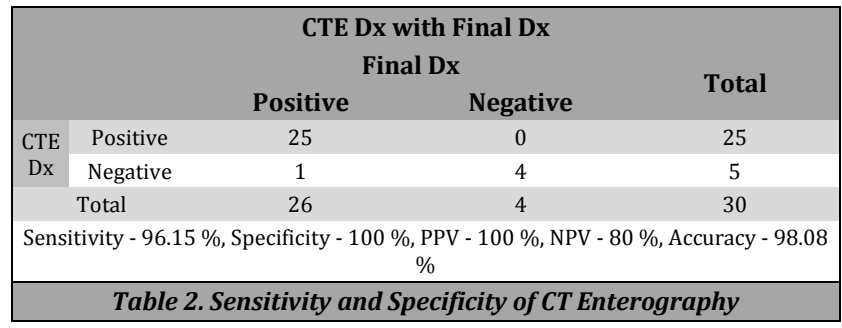

\section{DISCUSSION}

Small bowel can be affected by a wide spectrum of pathologies and is a challenging public health issue of considerable importance and magnitude in India. Various forms of involvement of the bowel are observed in the form of ITB, Crohn's disease, radiation enteritis, ischemic strictures etc.

The mean age of patients in our study was $40+/-16$ years. Out of 30 patients, females were $14(46.7 \%)$ and males were 16 (53.3\%). In our study abdominal pain was the most common complaint, present in all $30(100 \%)$ of the patients followed by anorexia seen in 26 (86.7 \%) cases. 15 (80 \%) patients presented with small bowel obstruction and about 4 (13.3\%) had a previous surgical history.

In our study, bowel thickening and mural hyperenhancement were the most common CT enterography findings, present in $23(76.7 \%)$ cases each, followed by significant lymphadenopathy was present in 12 (40\%) cases. Engorged vasa recta was seen in $10(33.33 \%)$ patients. Bowel was dilated in 10 (33.3\%) cases. Ascites was seen in 10 (33.3 \%) patients. Skip lesions were present in $6(20 \%)$ cases. Strictures were seen in $5(16.6 \%)$ cases. Findings are similar to previous studies. ${ }^{7}$

Intestinal pathologies were classified as either involving the jejunum, ileum or other colonic segments in isolation or in different combinations. IC junction was considered to be a part of the terminal ileum and cecum was considered to be a part of the large bowel for our study. In our study, ileum was the most commonly involved segment (47.73\%) which was in concordance with other studies. ${ }^{7,8}$

Mural thickening was defined as mural wall thickness exceeding $3 \mathrm{~mm}$. Mural thickening could be caused due to both non-neoplastic causes like ITB, Crohn's disease, ischemic strictures, radiation enteritis, ulcerative colitis etc. Neoplastic causes of thickening include adenocarcinoma, gastrointestinal stromal tumour (GIST), metastases and lymphoma. ${ }^{9}$ In our study, bowel wall showed increased thickening in 23 (76.7 \%) cases. Mural thickening was mostly caused by non-neoplastic conditions (91.3\%) with 2 cases (8.7\%) of neoplastic adenocarcinoma. Our findings are similar to previous studies. ${ }^{7}$ 8

Mural thickening was classified as mild $3-4 \mathrm{~mm}$ (infectious enteritis, mild Crohn's Disease), moderate 5-9 mm (Crohn's disease, early adenocarcinoma and lymphoma) and severe $>10 \mathrm{~mm}$ (Crohn's disease and neoplasms including lymphoma)..$^{9}$ In our study, among the 23 patients who showed thickening of the bowel wall, 18 (78.26\%) of the cases showed mild-moderate thickening while marked thickening was seen in $5(21.74 \%)$ cases. This was in concordance with a study by Megally et al. ${ }^{10}$ in which among the non-neoplastic group of patients, $81.8 \%$ cases presented with mild-moderate thickening. Both the neoplastic cases showed marked bowel 
wall thickening (100\%), while in study of Megally et al. ${ }^{10} 13$ of the 17 subjects $(76.5 \%)$ showed marked bowel wall thickening.

Bowel wall thickening that is asymmetric and irregular usually indicated malignant pathology while symmetrical and regular mural thickening is usually seen in benign pathologies but can also be seen in neoplastic pathologies. ${ }^{11}$ In our study, the type of wall thickening was symmetrical in 19 (82.61\%) cases; however, 4 (17.39 \%) patients presented with asymmetrical thickening. Both (100 \%) of the neoplastic cases and 2 of the patients with Crohn's disease showed asymmetrical thickening in our study. These findings were similar to other studies. ${ }^{10}$

In our study, among the cases with abnormal bowel wall enhancement majority (68.2\%) of the mural enhancement displayed a target pattern. Homogeneous enhancement was seen in $5(22.7 \%)$ cases with $2(9.1 \%)$ patients having heterogeneous mural enhancement. Both the patients that showed heterogeneous enhancement pattern had a neoplastic aetiology (adenocarcinoma). The patient with radiation enteritis and both the patients with ischemic strictures also had a target pattern of enhancement. This is similar to other studies. ${ }^{12}$

Pathologic conditions can cause focal involvement $(\leq 5$ $\mathrm{cm})$, segmental involvement $(6-40 \mathrm{~cm})$, or diffuse involvement $(>40 \mathrm{~cm}){ }^{13}$ Focal thickenings were mostly associated with malignancies (except lymphoma). Malignancies could present with asymmetric heterogeneous focal thickening. However certain inflammatory conditions could also present with focal mural thickening. Perienteric findings like predominant and out of proportion fat stranding in comparison with the focal bowel thickening would suggest inflammatory pathologies like appendicitis, diverticulitis, omental infarction. ${ }^{11}$ Segmental and diffused bowel thickenings were usually associated with benign conditions (except lymphoma) like infections, inflammation and ischemia. In our study majority ( $84 \%$ ) of the cases with bowel wall pathology showed segmental involvement. 2 (8\%) of the cases showed diffuse pattern of involvement with $2(8 \%)$ patients having focal pattern. In our study, both the cases having diffuse pattern of bowel involvement was seen in patients with ulcerative colitis where the colon was diffusely involved by the pathology. Focal involvement in our study was shown by both the patients with adenocarcinoma of the bowel. In another study by Chamail et al. ${ }^{14}$ both tuberculosis and adenocarcinoma caused focal thickening. Malignancy was associated with focal thickening in majority ( $54 \%$ ) cases. Out of the tubercular aetiology, majority (54\%) of the thickening was segmental.

In our study, majority $(76.67 \%)$ of the patients showed adequate Grade III distension of both the jejunal and ileal loops. Grade II distensibility of the jejunal loops and ileal loops were seen in $23.3 \%$ of the cases. In another study by Elamparidhi et al. ${ }^{15}$ while using mannitol as the contrast agent, $56 \%$ of the cases showed Grade II distensibility with $40 \%$ having distensibility of Grade III.

CT enterography was well tolerated by patients in our study. Nausea was the most common adverse effect noted being present in $5(16.7 \%)$ cases. Abdominal discomfort and post procedural discomfort was reported by $2(6.67 \%)$ patients each. 1 patient (3.3\%) reported an episode of diarrhoea after the investigation was performed. No other adverse experience was encountered as none of the patients' experienced vomiting. These findings are similar to other studies. ${ }^{15-17}$

In our study, mesenteric lymph nodes were present in 20 (66.7\%) cases, however only 12 (40\%) of them had significant lymphadenopathy. Lymph nodes were considered significant (lymphadenopathy) if they measured $>10 \mathrm{~mm}$ in short axis dimension. Mesenteric lymph nodes were present in $20(66.7 \%)$ cases, however only 12 (40\%) of them had significant lymphadenopathy. Calcification of the nodes was not present in any of the cases. However necrosis of the lymph nodes were seen in $7(23.3 \%)$ cases. $5(16.7 \%)$ patients presented with matting of the mesenteric lymph nodes. Fat stranding was seen in 18 (60\%) cases. Proliferation of fat in the surrounding mesentery was present in 7 (23.3\%) patients. Collection in the fat was noted in only $1(3.3 \%)$ of the case. Peritoneal and omental linings also showed abnormalities with a frequency of 7 (23.3\%) and 4 (13.3\%) cases respectively Free fluid was seen in the abdomen in one third $(33.3 \%)$ of the total cases.

In our study, CT enterography showed a sensitivity of $96.15 \%$ specificity $100 \%$, PPV $100 \%$, NPV $80 \%$ and an accuracy of $98.08 \%$ for the detection of small bowel diseases. This was in close concordance with a study by Masselli et al. ${ }^{18}$ which showed an overall sensitivity, specificity, and accuracy, respectively, in identifying patients with small-bowel lesions were $75.9 \%$ ( 41 of 54), $94.8 \%$ (91 of 96 ) and $88.0 \%$ (132 of 150) for CT enterography.

\section{CONCLUSIONS}

CT enterography is a quick and robust technique that is well tolerated by the patients. It provides excellent non-invasive depiction of small bowel pathologies, provides assessment of extra-intestinal and other abdominal organ pathology \& lung bases.

Data sharing statement provided by the authors is available with the full text of this article at jemds.com.

Financial or other competing interests: None.

Disclosure forms provided by the authors are available with the full text of this article at jemds.com.

\section{REFERENCES}

[1] Triester SL, Leighton JA, Leontiadis GI, et al. A metaanalysis of the yield of capsule endoscopy compared to other diagnostic modalities in patients with non stricturing small bowel Crohn's disease. American Journal of Gastroenterology 2006;101(5):954-64.

[2] Higgins PDR, Caoili E, Zimmermann M, et al. Computed tomographic enterography adds information to clinical management in small bowel Crohn's disease. Inflammatory Bowel Diseases 2007;13(3):262-8.

[3] Siddiki HA, Fidler JL, Fletcher JG, et al. Prospective comparison of state - of - the - art MR enterography and CT enterography in small - bowel Crohn's disease. American Journal of Roentgenology 2009;193(1):113-21. 
[4] Boriskin HS, Devito BS, Hines JJ, et al. CT enterography vs. capsule endoscopy. Abdominal Imaging 2009;34(2):14955.

[5] Harshad J, Nirad M, Devendra D, et al. Comparison of CT enterography with MR enterography and utility of MRI severity index, in Crohn's disease: a retrospective analysis. Tropical Gastroenterology 2019;39(3):117-23.

[6] Balthazar EJ, Gordon R, Hulnick D. Ileocecal tuberculosis: CT and radiologic evaluation. AJR American Journal of Roentgenology 1990;154(3):499-503.

[7] Misra RN, Bajaj S. Role of CT enterography in evaluation of small bowel disorders. Int J Res Med Sci 2019;7(2):53743.

[8] El-Kalioubie M, Ali R. Abdominal CT enterography as an imaging tool for chronic diarrhea: review of technique and diagnostic criteria. The Egyptian Journal of Radiology and Nuclear Medicine 2015;46(2):275-86.

[9] Ilangovan R, Burling D, George A, et al. CT enterography: review of technique and practical tips. The British Journal of Radiology 2012;85(1015):876-86

[10] Megally HI, Seifeldein GS, Abbas NA, et al. The diagnostic role of MDCT enterography in small bowel lesions. The Egyptian Journal of Radiology and Nuclear Medicine 2015;46(1):1-8.

[11] Fernandes T, Oliveira MI, Castro R, et al. Bowel wall thickening at CT: simplifying the diagnosis. Insights Imaging 2014;5(2):195-208.

[12] Varma RU, Rajpal KL, Bite NR, et al. Utility of CT enterography in the evaluation of small bowel pathologies. International Journal of Advances in Medicine 2017;4(5):1328.

[13] Macari M, Megibow AJ, Balthazar EJ. A pattern approach to the abnormal small bowel: observations at MDCT and CT enterography. American Journal of Roentgenology 2007;188(5):1344-55.

[14] Chamail A, Aggarwal N, Makhaik S, et al. CT enterography for diagnosis of small bowel disorders. JMSCR 2018;6(8):372-9.

[15] Elamparidhi P, Sivaranjanie S, Kumar RR, et al. Comparison of water, mannitol and positive oral contrast for evaluation of bowel by computed tomography. International Journal of Anatomy, Radiology and Surgery 2017;6(4):R013-R07.

[16] Prakashini K, Kakkar C, Sambhaji C, et al. Quantitative and qualitative bowel analysis using mannitol, water and iodine - based endoluminal contrast agent on 64 - row detector CT. The Indian Journal of Radiology \& Imaging 2013;23(4):373-8.

[17] Kolbe AB, Fletcher JG, Froemming AT, et al. Evaluation of patient tolerance and small - bowel distention with a new small - bowel distending agent for enterography. AJR American Journal of Roentgenology 2016;206(5):9941002.

[18] Masselli G, Di Tola M, Casciani E, et al. Diagnosis of small bowel diseases: prospective comparison of multidetector row CT enterography with MR enterography. Radiology 2016;279(2):420-31. 